\title{
Low Voltage X-ray Mapping: The Complementary Methods of the Oxford Instruments X-Max Extreme Windowless EDS Detector and the JEOL Soft X-Ray Emission Spectroscopy (SXES)
}

\author{
G. McMahon ${ }^{1}$, S. Burgess ${ }^{2}$, M. Takakura ${ }^{3}$, H. Takahashi ${ }^{3}$ and M.G. Burke ${ }^{1}$ \\ 1. Materials Performance Centre and Electron Microscopy Centre, University of Manchester, Manchester \\ UK \\ 2. Oxford Instruments NanoAnalysis, High Wycombe UK \\ 3. JEOL, SA Business Unit, JEOL Ltd., Akishima Tokyo Japan
}

As demands for higher spatial resolution in X-ray spectrum imaging increase, necessitating the use of lower electron beam accelerating voltages, new X-ray detection technologies have recently been introduced with the aim of optimizing X-ray acquisitions. Two such technologies are the Oxford Instruments X-Max Extreme Windowless Silicon Drift Detector (SDD) incorporating a $100 \mathrm{~mm}^{2}$ active detector area, and the JEOL soft X-Ray Emission Spectrometer (SXES). The former is a novel EDXS detector design, the latter a novel WDS detector design, and as such the two methods are quite complementary, each with its own set of advantages.

Here we compare the performance of the two technologies and highlight their unique capabilities and strengths using the same metallographic sample - a hot isostatically-pressed sample, which is a duplex stainless steel with roughly equal parts of austenite and ferrite, some $\mathrm{Nb}(\mathrm{Ti}) \mathrm{C}$ carbides, $\mathrm{M}_{7} \mathrm{C}_{3}$ carbides and an Fe-Cr-Ni silicide phase. The SXES analyses were performed on a JEOL 7800F FEG-SEM, whereas the EDXS analyses were conducted on a Zeiss Merlin FEG-SEM.

One of the unique advantages of the SXES detector is its extremely high energy resolution of $0.3 \mathrm{eV}$, as compared to values of 30-40 eV for the Extreme at the same X-ray energy. Not only does this enable separation of nasty X-ray line interferences (eg. Ti-L and N-K) as demonstrated in Figure 1, but also allows for chemical state mapping. Analysis of three different areas in the $\mathrm{Nb}(\mathrm{Ti}) \mathrm{C}$ yielded significantly different peak distributions of the second order Ti-Ll and N-K lines. Regions of interest (ROI) can be defined at each of the shoulders (Figure $1 \mathrm{~b}$ as well as for the second order C-K line in Figure 1c), and the area of interest mapped for $\mathrm{N}, \mathrm{C}, \mathrm{Nb}$ and the chemical state of Ti. The result is shown in Figure 1d, where comparison of the bottom three images illustrates a difference in the concentration of $\mathrm{N}$ and the chemical state for Ti traversing the interior to the exterior of the carbide particle.

On the other hand, the X-Max Extreme EDXS detector performs remarkably well at low voltages, yielding count rates at low working distances, where one needs to operate for highest spatial resolution SEM imaging at low voltage. The X-ray spectrum images shown in Figure 2 display excellent spatial resolution, revealing features easily less than $100 \mathrm{~nm}$ in size (eg. the thin C-rich film on the edge of one of the $\mathrm{Nb}(\mathrm{Ti}) \mathrm{C}$ "florettes" or the nano-sized $\mathrm{Nb}$-rich precipitates evident in the $\mathrm{Nb}$ spectrum image. The importance of the high count rate cannot be under emphasized. Acquiring spectrum images quickly at low voltages not only minimized the effect of specimen drift, but also minimizes one of the bigger problems with low voltage X-ray spectrum imaging, the build-up of the ubiquitous hydrocarbon layer under the beam. The use of some form of anticontamination is quite important, be it a plasma cleaner, a liquid nitrogen cold finger, or both. 
(a)

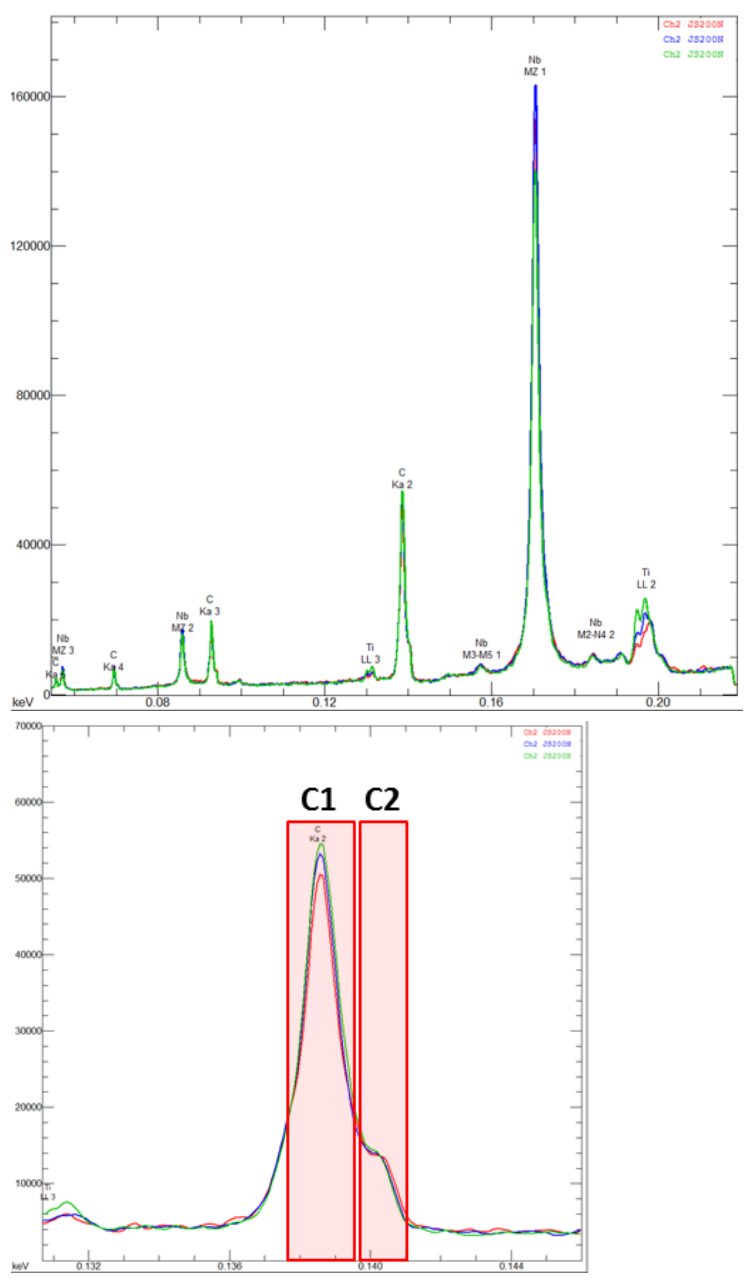

(b)

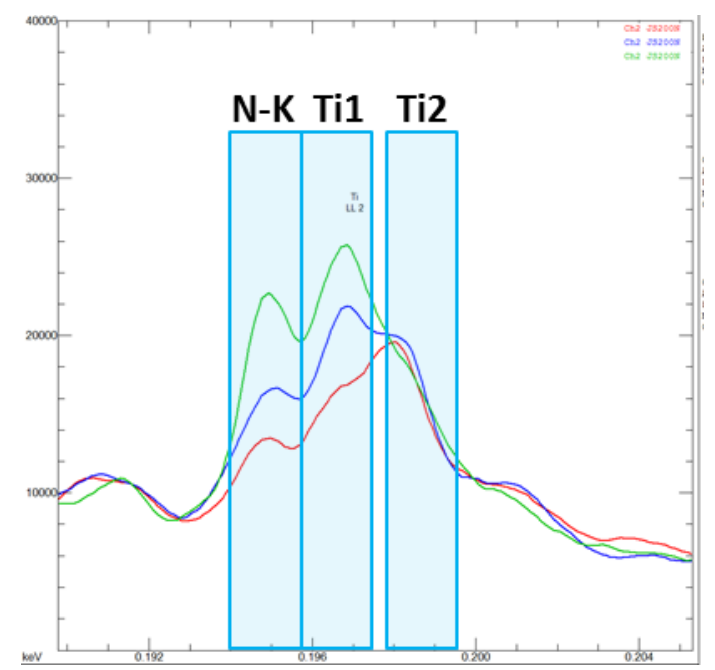

(c)

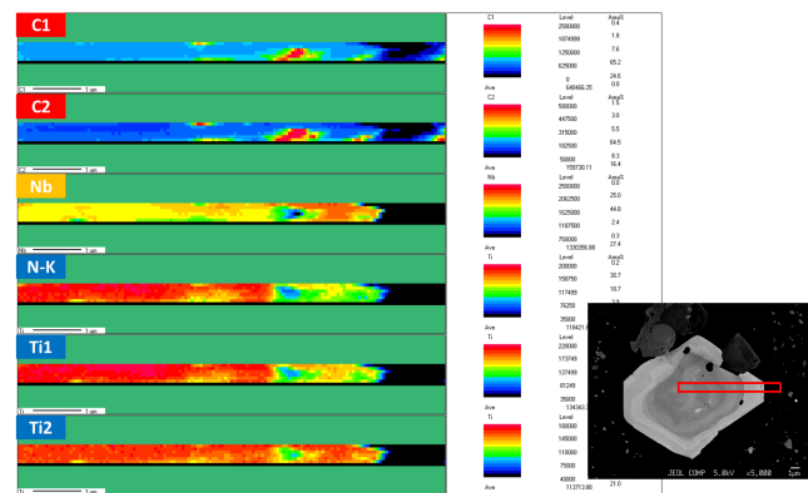

(d)

Figure 1. (a) SXES spectrum from area within $\mathrm{Nb}(\mathrm{Ti}) \mathrm{C}$ grain. (b) and (c) ROI definition for mapping of $\mathrm{C}$ and $\mathrm{N}$ and chemical state mapping of Ti. (d) SXES maps from area shown in backscatter image (inset) for $\mathrm{Nb}, \mathrm{Ti}, \mathrm{C}$ and $\mathrm{N}$. Beam current is $50 \mathrm{nA}$.

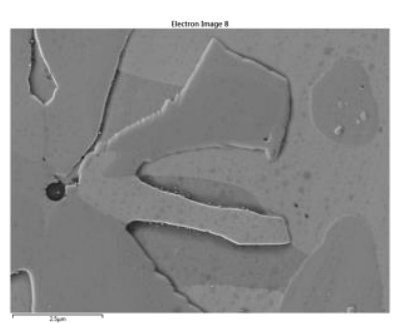

$\mathrm{Nb} \mathrm{M \zeta}$

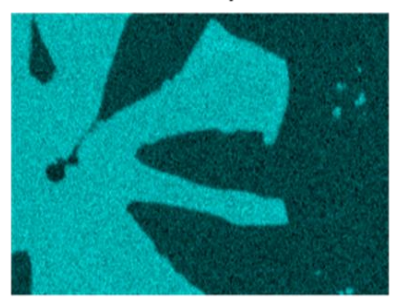

$2.5 \mathrm{~m}$
Fe L $\alpha 1 \_2$

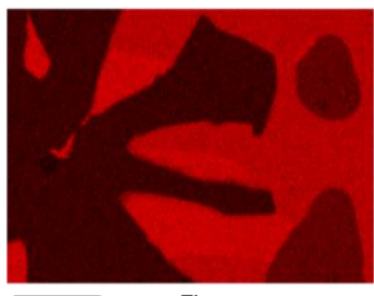

$\longdiv { 2 . 5 \mu \mathrm { m } } \mathrm { Ti }$

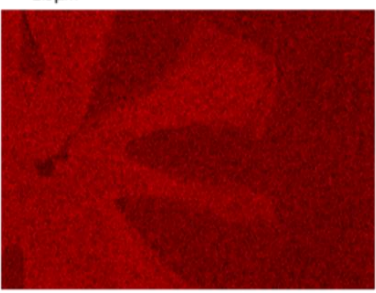

2.5um
C Ka1_2
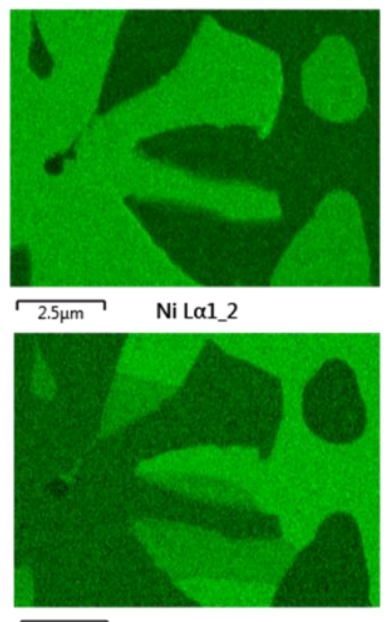

$2.5 \mu m$

Figure 2. EDX spectrum images acquired at $2 \mathrm{kV}$ and $2.2 \mathrm{nA}$ beam current. 